\section{Leistungen und Grenzen der Thermodynamik in der Umweltökonomie}

\begin{abstract}
Man nehme zwei Behälter mit Flüssigkeiten unterschiedlicher Temperatur und stelle sie eng nebeneinander. Dann isoliere man sie gut gegen äußere Einflüsse, nachdem man sie jeweils mit einem Thermometer versehen hat. Nun setze man sich vor die beiden Meßinstrumente, und schon nach einiger Zeit wird man feststellen, daß sich die Temperaturen annähern. Dieser Prozeß wird solange fortschreiten, bis die Temperaturen gleich sind. Jetzt werden wir keine Temperaturveränderungen mehr wahrnehmen; wir können getrost aufstehen und gehen in der Sicherheit, daß sich nichts mehr ändern wird. Denn die Experimentanordnung befindet sich in einem sogenannten thermodynamischen Gleichgewicht. Dieses Ergebnis beunruhigt uns nicht weiter, denn wir haben uns im Alltag daran gewöhnt. Daher kämen wir auch nie auf die Idee, das Experiment umzudrehen, zwei Behälter mit gleicher Temperatur nebeneinander zu stellen, diese von der Umwelt zu isolieren, und zu warten, bis die Temperaturen divergieren. Wir wissen, daß dieser thermodynamische Prozeß nur in einer Richtung abläuft, daß er irreversibel ist, wie die Naturwissenschaft es ausdrückt.
\end{abstract}

Gewöhnlicherweise beunruhigt uns das Ergebnis dieses Experiments in keiner Weise. Und doch sind Naturwissenschaftler, wie etwa Lord Calvin, durch diese oder ähnliche Beobachtungen zu pessimistischen Folgerungen in Hinblick auf das Leben auf Erden gelangt. Lord Calvin sprach z. B. vom Wärmetod, den das Universum über kurz oder lang ereilen würde. $\mathrm{Zu}$ dieser pessimistischen Vision gelangten er und auch viele andere Naturwissenschaftler, da die thermodynamischen Gesetze, die in diesem Experiment zum Ausdruck gelangen, sich auch auf alle anderen Energieformen, die für uns lebenswichtig sind (chemische, elektrische, mechanische Energie), beziehen. Früher oder später - die Geschwindigkeit dieses Prozesses wird auch durch die Aktivitäten der Menschen bestimmt - wird alle Energie in Wärme gleicher Temperatur umgewandelt. Der Thermodynamiker nennt diesen lebensfeindlichen Endpunkt einen Zustand maximaler »Entropie «. Der Endpunkt ist lebensfeindlich, da nunmehr kein Energieaustausch mehr stattfindet. Und wo kein Energieaustausch stattfinden kann, da fehlt auch die energetische Grundlage für das Leben.

\section{Der Begriff Entropie}

Was ist nun $»$ Entropie $\ll$ ? Der Begriff Entropie wird in der Thermodynamik verwendet und bezeichnet jene Menge, die im Rahmen eines Wärmeenergieaustausches zwischen zwei Systemen fließt. Unsere beiden Systeme sind die beiden Flüssigkeitsbehälter, zwischen denen ein Wärmeenergieaustausch stattfindet. Da unsere experimentelle Anordnung gegen Außeneinflüsse ab- geschirmt ist, bleibt die Gesamtenergie daher dieselbe. Das weiß man wegen der Gültigkeit des ersten thermodynamischen Hauptsatzes, des Energieerhaltungssatzes. Energie entsteht nicht oder wird auch nicht vernichtet, sie wird nur ausgetauscht und zwar nicht abstrakt, sondern nur in meßbaren Energieformen, also beispielsweise durch Bewegung (Impuls), Kompression (Volumen), Elektrizität (Ladung), chemische Energie (Teilchenzahlen) oder eben Wärmeenergie (Entropie). Entropie kann man also messen, so wie man Volumen, Impulse, elektrische Ladung usw. messen kann.

Den Energieerhaltungssatz können Ökonomen sehr gut nachvollziehen. Besagt er doch, daß in einem geschlossenen System die Summe aller Zuflüsse der Summe aller Abflüsse jeden Subsystems entsprechen müssen. Das ist das grundlegende Prinzip, auf das die doppelte Buchführung aufbaut. Das Prinzip der Buchung und Gegenbuchung sichert, daß man nicht versehentlich in ein geschlossenes System Zu- oder Abflüsse hinzurechnet. Soweit gibt es eine große Ähnlichkeit zwischen Thermodynamik und der Kreislaufbetrachtung der Makroökonomie (auch Thermodynamiker sprechen von der Bilanzierung der Energie). Was aber Ökonomen nicht so einleuchtend erscheinen mag, ist der Tatbestand, daß ein System (hier: unsere beiden Flüssigkeitsbehälter) auf dem Wege zu einem thermodynamischen Gleichgewicht Entropic erzeugt. Entropie ist eine meßbare Menge, und man mag sich fragen, wieso in einem geschlossenen System eine Menge zunehmen kann. Dies liegt einfach daran, daß im Gegensatz zu anderen mengenmäßigen physikalischen Größen - der Erhaltungssatz für die Entropie nicht gilt. Das zeigt sich einfach in unserem Experiment. Der Erhaltungssatz bezieht sich auf die Energie von Wärme, nennen wir sie W, und diese wird nicht nur vom Entropiestrom, sondern auch von der Temperatur bestimmt. Es gilt $\mathrm{W}=\mathrm{T} \times \mathrm{dS}$, wobei $\mathrm{T}=$ Temperatur. In Lehrbüchern der Thermodynamik wird unter Zuhilfenahme dieser Beziehung in einigen wenigen algebraischen Schritten gezeigt, daß der oben beschriebene Temperaturangleich mit einer Zunahme an Entropie verbunden ist (1).

\section{Implikationen für Ökologen und Umweltökonomen}

Inwieweit ist nun dieses kleine Experiment und seine thermodynamischen Implikationen für Ökologen und Umweltökonomen relevant? Zunächst einmal fällt auf, daß die Rahmenbedingungen des Experiments und die der real existierenden Welt (Erdkugel) nicht die gleichen sind. Wir stehen in energetischem Austausch mit der Sonne (Der Energiefluß zwischen Sonne und Erde beträgt etwa $1.7310^{14} \mathrm{~kW}$ ). Unser System ist also nicht isoliert sondern nur »abgeschlos- sen«, d. h., Energieaustausch ist möglich, nicht jedoch Massenaustausch. In diesem Fall gilt nur der Erhaltungssatz der Masse, der besagt, daß in einem isolierten System ein Zuwachs oder Abbau an Materie nicht denkbar ist.

Den Erhaltungssatz der Masse hat sich der sog. Materialbilanzansatz in seiner inzwischen ausdifferenzierten Systematik zu eigen gemacht (2). Das ökonomische System wird nicht mehr als ein geschlossenes System angesehen, sondern die Bilanzidentitäten werden auf das Gesamtsystem Umwelt und Wirtschaft bezogen. »Natur«bzw. »Umwelt « werden nun in die umweltökonomische Betrachtung als weiterer Pol eingeführt, nicht nur als Ressourcenreservoir, sondern auch als Absorbtionsmedium des durch das ökonomische System bedingten Materialrückflusses (Abfall). Denn das erzwingt die naturwissenschaftliche Sichtweise des ökonomischen Systems: Jedem Ressourcenverzehr steht dieselbe Menge an »Natureintrag « durch das ökonomische System gegenüber. Im Materialbilanzansatz wird also die volkswirtschaftliche Gesamtrechnung durch ein differenziertes Kontensystem der Natur ergänzt, wobei die Maßeinheiten nicht mehr monetär, sei es nominell oder real, sondern phsikalisch, d.h. Masseeinheiten (Tonnen, kg, Mol, usw. ), sind.

\section{Zwang zur ganzheitlichen Betrachtung}

Dieser Ansatz zwingt also Umweltökonomen zur ganzheitlichen Betrachtung. Es gibt kein isoliertes Ressourcenproblem und auch nicht ein isoliertes Abfallproblem, sondern das eine bedingt das andere. Die Berücksichtigung des Erhaltungssatzes der Masse in der Umweltökonomie zwingt $\mathrm{m}$. E. die Umweltökonomen viele herkömmliche Ressourcenmodelle tiefgreifend zu modifizieren. Unsere Lebensqualität hängt nicht nur vom Ressourcenverzehr durch Konsum, sondern auch vom produzierten Abfall, jenem lästigen Kuppelprodukt der Produktion, ab. Dabei stelle man sich unter Abfall nicht nur eine amorphe und auch teilweise gesundheitschädigende Mischung in den Mülleimern der Haushalte oder den Containern der Industrie vor, sondern auch solche wohlstrukturierte Konsumartikel, die durch die (massen)psychologisch bedingte Änderungen der Sinneswahrnehmung (Mode, technischer Fortschritt) als abgeschrieben gelten können. Was also Abfall ist, ist nicht nur eine biologische Frage der gesundheitlichen Verträglichkeit, sondern auch kulturell bedingt.

Das alles wäre recht harmlos, und bedürfte auch keiner tieferen Betrachtung, wenn die Gigatonnen an ehemals nutzenstiftenden Gütern ohne große Aufwendungen in den Produktionsprozeß zurückgeschleußt und ohne großen Ressourcenverzehr jenen kulturellen »back-up» erfahren könnten (Recycling), der sie wieder konsumfähig macht. Oder aber wir könnten auf die Resorbtions- und Reinigungsfunktion der Natur setzen, die diese Güter nicht nur zersetzt, sondern auch in neue, wiederverwendbare Ausgangstoffe umwandelt. Der Materialbilanzansatz wäre dann 
recht überflüssig. Er wäre dann nur das analytische Raster, mit dem ein gefälliger Blick auf das Raumschiff $»$ Erde« geworfen werden könnte. Das Zusammenwirken von Ökonomie und Umwelt könnte dann als harmonisches Ganzes betrachtet werden, das auf beliebigen Konsumniveaus stabilisiert werden könnte.

\section{Ungedeckte Absorbtionsnachfrage der Weltökonomie}

Aber genau darauf können wir eben nicht mehr setzen, nämlich, daß das Absorbtionspotential der Natur die Absorbtionsnachfrage der Weltökonomie deckt. Dies ist Ökologen und auch Umwelt- und Ressourcenökonomen schon sehr lange bekannt. Es gibt eine fast nicht mehr überschaubare Zahl an neoklassischen Umweltmodellen, die die begrenzte Resorbtionsfähigkeit der Natur berücksichtigen. Auch Umweltlehrbücher weisen inzwischen darauf hin, daß ihre präsentierten Modelle der optimalen Bewirtschaftung regenerierbarer Ressourcen durch einfache Umdefinition der Variablen auch für das optimale Management der Assimilationsfähigkeit der Natur verwendbar werden.

Wozu bedarf es aber dann des Entropie-Ansatzes? Ist denn das Problem der Übernutzung der natürlichen Umwelt nicht ausreichend in den Modellen der herkömmlichen Umweltökonomie berücksichtigt? Es gibt eine kleine Gruppe an Umwelt- und Ressourcenökonomen und auch Ökologen, die jene wohlfahrtstheoretischen $\mathrm{Pa}$ radigmen für obsolet halten und für eine stärkere Einbindung von physikalischen und biologischen Ansätzen in die Umweltökonomie plädieren. Ihr Ziel ist wohl eine übergreifende »ÖkosystemTheorie«. $\mathrm{Zu}$ dieser Gruppe gehören sicherlich auch die $»$ Thermodynamiker «, die ich in drei Gruppen einteilen möchte.

Die erste Gruppe besteht aus den Verfechtern des Materialbilanzansatzes, deren Hauptanliegen wohl darin liegt, den Erhaltungssatz der Thermodynamik für die Umweltpolitik operationalisierbar zu machen. Unter Verwendung der InputOutput-Analyse haben sie statisch und dynamisch Strukturmodelle entwickelt, die auf verschiedenen Ebenen (international, regional, betrieblich) einsatzfähig sind. Insbesondere die Vielzahl an Regionaluntersuchungen und die Ausarbeitung der Produktlinienanalyse zeigt an, daß sich dieser Ansatz als analytischer Rahmen für Umweltpolitik sehr gut eignet. Dieses Forschungsprogramm verwendet seine zeitlichen und budgetären Kapazitäten für die Ermittlung von Interdependenzzusammenhängen zwischen Umwelt und Industrie und scheint mir in Kombination mit dem Preis-Standard-Ansatz ein umweltpolitisch schlagkräftiges und gleichzeitig analytisch komplexes aber auch transparentes Konzept zu sein. Die monetäre Evaluierung von Umweltschäden, wie sie in vielen wohlfahrtstheoretisch orientierten Kosten-Nutzen-Analysen der neoklassischen Umweltökonomie betrieben wird, ist diesem Ansatz fremd. Der Material- bilanzansatz ist $\mathrm{m}$. E. ein wichtiger Baustein für eine ökonomische Theorie der Umwelt. Ihm verdanken wir die Weiterentwicklung der Input-Output-Rechnung, mit der die Interdependenz zwischen Umwelt und Wirtschaftssystemen klassifiziert und einer wissenschaftlichen und umweltpolitischen Öffentlichkeit nahe gebracht werden kann.

Die zweite Gruppe, die man »thermodynamische Pessimisten« nennen könnte, problematisiert die Ressourcenverfügbarkeit, indem sie die optimistische Annahme der Existenz von regenerierbaren Ressourcen in Frage stellt. Energieaustausch ist ja an Energieformen gebunden, so auch an die in den Ressourcen lagernde Energie (Energieträger). Aber nicht nur der Energieaustausch, sondern auch die Transformation von Masse (biologischer, chemischer Masse) ist eine unabdingbare Voraussetzung für Leben (man denke etwa an die Kohlenstoffkreisläufe). Gelten hier auch die pessimistischen Prognosen der frühen Thermodynamiker? Steht uns früher oder später der Tod in einer amorphen, nicht mehr strukturierten und damit nicht mehr verfügbaren Masse bevor?

Dieses Szenario läßt sich durch den statistischen Entropiebegriff, durch ein ähnliches Experiment, wie oben beschrieben, erläutern. Die beiden Behälter seien jetzt mit zwei unterschiedlichen Gasen gefüllt. Man stelle sie neben einander und achte darauf, daß keine weiteren Moleküle von aưßen eindringen können. Dann entferne man die benachbarten Trennwände. Die Moleküle beider Gase, nennen wir sie Rot-Gas und Gelb-Gas, können sich nun beliebig mischen. Wo werden sie denn hauptsächlich anzutreffen sein? Im linken oder im rechten Behälter? Das kann man nicht genau angeben, man kann nur Wahrscheinlichkeiten der räumlichen Häufung angeben und die Wahrscheinlichkeit, daß Rot und Gelb gleichverteilt sind, ist am größten. Das ist das Maximum an Entropie im statistischen Sinne. Denn zu diesem Zustand wird unser System, die beiden zusammengefügten Behälter, hinstreben, d.h. das wird der am häufigsten beobachtete Zustand sein. Wir haben die Behälter nicht energetisch von Außeneinflüssen isoliert. Die Rahmenbedingungen weisen also eine größere Ähnlichkeit mit der Situation unserer Erde auf, das System ist nicht isoliert, sondern nur abgeschlossen.

\section{Der 4. Hauptsatz der Thermodynamik}

Der Physiker und Ökonom Georgescu-Roegen behauptet nun, daß auch für abgeschlossene Systeme der zweite Hauptsatz der Thermodynamik per Analogie gelte. Das ist sein 4. Hauptsatz der Thermodynamik. Auf unser Experiment angewandt: Das System strebt im Laufe der Zeit zu einem Zustand der unstrukturierten Gleichverteilung (sog. »mixed-upness«), einem Zustand größter Unordnung, denn der Mangel an Struktur, also an strukturierten Häufungen (etwa: Rot bleibt im linken, Gelb im rechten Behälter) ist unwahrscheinlich. Deklarieren wir die Menschheit

\section{Neues Jabr, neues Gesicht!}

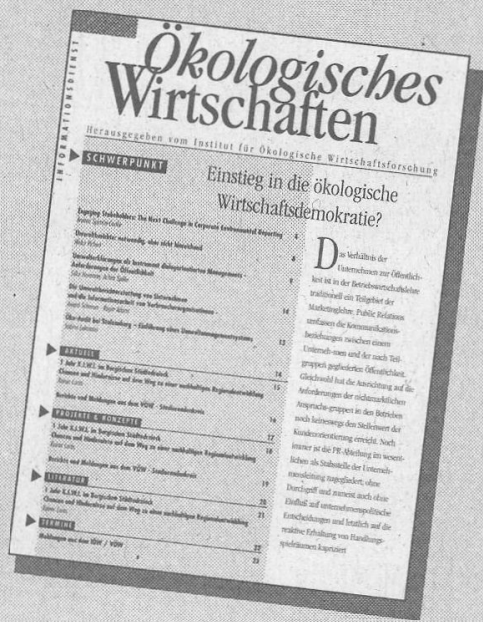

Ab 15. Februar 1996 präsentiert sich der IÖW/VÖW Informationsdienst unter dem Titel

„Ökologisches Wirtschaften"

in einem neuen Gewand. Geändertes Layout, übersichtlichere Grafiken, größere Schrift und andere Farbwahl sind nur einige Optimierungen, die Sie erwarten.

Für das neue Layout zeichnet die Gesellschaft für ökologische

Kommunikation, München, kurz: ökom-Verlag verantwortlich.

Das ökom-Team - bekannt durch die Zeitschrift „Politische Ökologie“ übernimmt in Zukunft den Verlag und Vertrieb des Informationsdienstes.

Inhaltlich bleibt jedoch alles beim alten: Der Informationsdienst „Ökologisches Wirtschaften" ist nach wie vor das Organ von IÖW und VÖW.

\footnotetext{
Geben Sie also in ökom GmbH Zukunft Ihre Waltherstr.29, Rgb. Bestellungen der 80337 München ökom GmbH - Tel.: 089/544 184-0 bekannt. Fax: 089/544 184-99
} 
zu den Pförtnern der Trennwand, so bedeutet dies: Der menschliche Ressourcenverzehr (Verwendung von Gelb und Rot) und die Rückführung amorpher Abfälle (Rückführung eines Gemisches von Gelb und Rot in beide Behälter) in die Umwelt führt langfristig in einen amorphen Zustand der Natur, einer Anhäufung von unstrukturierter Masse, aus der eine Ressourcenextraktion der reinen Ausgangsstoffe (Gelb, Rot) mit einem Energiebedarf verbunden ist, der durch die von außen eindringenden Energie nicht mehr gedeckt werden kann. Auf uns wartet nicht der Hitzetod, sondern wir beschreiten den Sterbeweg durch Produktion von Unordnung.

Gegen Georgescu-Roegen's 4. Hauptsatz können verschiedene Einwände vorgebracht werden, auch angesichts sinkender Rohstofflager und steigender Müllberge. Erstens kann nicht ohne Beweise ausgeschlossen werden, daß die von außen einströmende Energie für die Ressourcenextraktion aus amorphen Energieträgern doch ausreicht; zweitens ist a priori nicht geklärt, welche Rohstoffe für den Menschen wertvoll sind und damit immer verfügbar sein müssen -dies hängt ja auch von der Adaptionsleistung dieser Gattung $\mathrm{ab}-;$ drittens führt diese pessimistische Diagnose in eine Aporie, denn sie kann nicht erklären, wie Ordnung enstanden ist (3), und viertens spricht die Evolution differenzierten Lebens gegen dieses Gesetz.

Damit kommen wir zur dritten Gruppe, die das Konzept der $»$ thermodynamischen Effizienz« geprägt haben. Leben, sei es individuell oder das einer Population, wird als ein Evolutionsprozeß betrachtet, der sich durch eine Zunahme an Komplexität auszeichnet. Dies gilt nicht nur für biologische Zusammenhänge, sondern auch für soziale, psychologische und ökonomische Zusammenhänge. Diese Zunahme an differenzierter Struktur wird durch die Versorgung mit Ressourcen und durch die Möglichkeit der Abgabe von unstrukturierten Abfällen an die Umwelt ermöglicht. Die lebendigen Systemeinheiten können als »dissipative Strukturen « (Prigogine [4]) bezeichnet werden, deren Stoffwechsel thermodynamisch betrachtet nichts anderes als Schaffung niedriger Entropie im Inneren durch Abgabe hoher Entropie an die Außenwelt ist. Im Kontext unseres statistischen Entropiebegriffs heißt dies, daß Leben die Aufnahme strukturierter Materie und Abgabe unstrukturierten Abfalls voraussetzt.

Die Tatsache, daß Leben existiert und die Evolution eine Bewegung zu komplexeren Strukturen aufweist, deutet offenbar darauf hin, daß die natürliche Welt durch Aufnahme solarer Energie in der Lage ist, sich langfristig vom tödlichen thermodynamischen Gleichgewicht entfernt zu halten, ja sich jenseits des Gleichgewichts zu stabilisieren. Diese Stabilisierung wird durch einen Selbststeuerungsmechanismus der Systeme erreicht. Durch Aufnahme von ökologischer Information und ihrer Umsetzung in entsprechende Organisations- und Verhaltensmuster werden die Systeme thermodynamisch effizient, d. h. sie mi- nimieren die »spezifische Entropieproduktion «. Unter diesem Begriff versteht man die Entropieabgabe pro Einheit Biomasse.

Die Nicht-Gleichgewichts-Thermodynamik und ihre Bedeutung für die Umweltökonomie

Inwiefern ist nun die Nicht-GleichgewichtsThermodynamik für die Umweltökonomie von Bedeutung? Die Bewertung der Relevanz dieser Theorie für die Umweltökonomie hängt m.E. hauptsächlich davon ab, welche Verhaltensannahmen man trifft. Im Kontext der herkömmlichen, neoklassischen Ressourcentheorie (regenerierbare Ressourcen) scheint mir das Entropiekonzept überflüssig zu sein. Wenn die Annahme des »homo oeconomicus « aufrechterhalten wird, und der Allokationsprozeß als Ergebnis der Entscheidung von rationalen und wohlinformierten Individuen aufgefaßt wird, so führt die Berücksichtigung des Umweltproblems im Rahmen dieser Modelle dazu, daß die Naturressourcen effizient eingesetzt werden, sei es von selbst durch einen funktionierenden Marktmechanismus oder durch umweltpolitische Maßnahmen, die Verwaltung, Politik und Wissenschaft in steter Kooperation entwickeln und den sich wandelnden Rahmenbedingungen anpassen. Dies gilt sowohl für die wohlfahrtstheoretischen Ansätze, wie auch für den Preis-Standard-Ansatz. Das Konzept der »Stabilisierung eines Systems fern vom thermodynamischen Gleichgewicht« kann für das ökonomische System keine-weiteren Einsichten erbringen, solange die Ressourcenbewirtschaftung die Naturkreisläufe beachtet, die sich durch die Regenerationsfunktionen der Modelle ausdrüickt. Die Beschäftigung mit der nichtgleichgewichtigen Thermodynamik hat für den Umweltökonomen nur pädagogische Funktion, sich der komplexen Einbettung von Subsystemen (z.B. Wirtschaftskreisläufe) in einen ökologischen Kreislauf bewußt zu werden.

Das Konzept der Stabilisierung von thermodynamischen Ungleichgewichten gewinnt $\mathrm{m}$. E. erst dann eine Bedeutung für umweltökonomische Zusammenhänge, wenn die Genese und Evolution von Systemen nicht als Ausdruck rationaler individueller Entscheidungen archimedischer Umweltpolitiker angesehen wird. Wird die Aufnahme von Information, die Bildung von Präferenzen und technologischen Systemen, das Lernen und die Bildung von ökonomischen Entscheidungen selbst als evolutorischer Prozeß aufgefaßt, dann gewinnt der Begriff der thermodynamischen Effizienz eine große Bedeutung für die Umweltökonomie. Wenn die Systemsteuerung des Stoffwechsels mit der Natur selbst von dieser Steuerung wieder abhängig ist, dann sind biophysikalische Modelle der neueren Thermodynamik für die Abbildung dynamischer umweltökonomischer Prozesse hilfreich. Biomathematische Modelle finden bereits in der Soziologie und auch in der Industrieökonomie ihre Anwendung (5). In Modellen dieser Art wird geprüft, welche Verhaltensmuster sich im Zeitablauf im Rahmen eines Prozesses der Selektion durchsetzen, und zu welchem Zustand ein durch diese Verhaltensmuster determiniertes System konvergiert. Betrachtet man den Prozeß der Selbstregulierung von Systemen als endogenen Prozeß, so ist ihr Stoffwechsel mit dem gesamten ökologischen System (Entropieabgabe) innerhalb eines ökologisch-ökonomischen Modells zu erklären. Hier scheint mir der Rekurs auf dynamische biochemische Modelle in Kombination mit verhaltensevolutorischen Ansätzen vielversprechend zu sein.

Georg Meran, Berlin

\section{Anmerkungen}

1) Siehe z. B. Falk und Ruppel, S. 192 ff.

2) Zusammenfassend siehe z. B. Ayres, U. und U. Simonis.

3) Siehe hierzu die kosmologischen Beiträge in Weber et al.

4) Eine genauere Darstellung dieser Theorie kann man im Sammelband von Beckenbach und Diefenbach finden.

5) Siehe hierzu R. Selten (1991).

\section{Literatur}

- Ayres, R., Simonis, U. (1994): Industrial Metabolism: Restructering for Sustainable Development. United Nations University Press.

- Beckenbach, F., Diefenbacher, H. (1994): Perspektiven einer ökologischen Ökonomie. Marburg.

- Falk, G., Ruppel, W. (1976): Energie und Entropie. Eine Einführung in die Thermodynamik. Berlin-Heidelberg-New York.

- Selten, R. (1991): Evolution, Learning and Economic Behaviour. Games and Economic Behaviour 3, 3-24.

- Weber, H. et al. (1990): Entropy, Information, and.Evolution. Cambridge (M. I. T. Press).

Bei Bestellung von mehr als 20 Exemplaren dieses Themenheftes Ecological Economics z. B. für Lehrveranstaltungen werden pro Heft DM 3,--plus DM 10,-an Bearbeitungsgebühr pro Bestellung berechnet.

Bestellungen gegen Rechnung richten Sie bitte an die IÖW-Geschäftsstelle in Berlin. 
(c) 20I0 Authors; licensee IÖW and oekom verlag. This is an article distributed under the terms of the Creative Commons Attribution Non-Commercial No Derivates License (http://creativecommons.org/licenses/by-nc-nd/3.o/), which permits unrestricted use, distribution, and reproduction in any medium, provided the original work is properly cited. 